\title{
REPRESENTATION OF ORIENTATIONS OF SYMMETRICAL OBJECTS BY RODRIGUES VECTORS
}

\author{
P. NEUMANN \\ Max-Planck-Institut für Eisenforschung GmbH, D-4000 Düsseldorf, \\ F.R.Germany
}

\section{INTRODUCTION}

Orientation distribution functions, ODFs, are usually represented graphically in the Euler angle space, in spite of serious deficiencies, like a threefold representation of each orientation and a degeneracy of the invariant volume element near $\Phi=0$. Frank ${ }^{1}$ with his excellent article at the precessor of this conference has vividly argued in favour of the Rodrigues representation of rotations. It is given by

$$
\underline{r}=\tan (\omega / 2) \cdot \underline{n}
$$

where $\omega$ is the angle and $n$ the axis of a rotation. It is the purpose of this article to extend the arguments given by Frank and illustrate by some graphic examples the superior features of this representation.

\section{ANGLE AXIS REPRESENTATIONS OF THE FORM (1) HAVE NO SINGULARITY AT THE ORIGIN.}

Occasionally the opinion is expressed, that angle axis representations are ill-behaved at the origin and should be rejected on this ground. This is true only if the direction of the axis, $\underline{n}$, is given in polar coordinates by two angles, say $\phi$ and $\theta$. If this is done, the identity, E, is represented not by a point, but by the plane: $\omega=0$, arbitrary $\phi$ and $\theta$. Because of this singularity at $\mathbf{E}$ this particular angle axis representation has rightfully not gained much attention.

Let $f(\omega)$ be a monotonous function of the rotation angle with $f(0)=0$. If the cartesian coordinates of the vector $f(\omega) \underline{n}$ are used, $\mathbf{E}$ is represented by just one point, $(0,0,0)$, and no problems arise near $\mathbf{E}$. It will become clear by equation (13) that the opposit is true: the deviations from the ideal metric tend to zero when $\mathbf{E}$ is approached.

\section{THE EFFECT OF LATTICE AND SAMPLE SYMMETRIES ON THE ANGLE AND AXIS OF THE ROTATIONS}

Let $L_{i}$ be the transformation matrices of the lattice symmetries and $S_{k}$ those of the sample symmetries. If we describe the orientation of the lattice with respect to the sample by the transformation matrices between a sample and a lattice coordinate system, and if $O$ is one of them, then all of these matrices are given by

$$
\mathrm{O}_{\mathrm{ik}}=\mathrm{L}_{\mathrm{i}} \cdot \mathrm{O} \cdot \mathrm{S}_{\mathrm{k}}
$$


As an important special case we also consider the orientation of the lattice with respect to a given coordinate system (a "sample" without symmetry, $S_{k}=E$ ). This full orientation is obviously given by the following set of matrices

$$
O_{i}=L_{i} \cdot O
$$

Usually, the proper rotation with the smallest angle is picked from these for representing the full orientation. For cubic lattices (3) contains 24 matrices from which the selection can be made. In this way the angle can be kept always smaller than $62.8^{\circ} 2$. If mutual orientations of two cubic lattices are considered, we have $\left\{S_{k}\right\}=\left\{L_{i}\right\}$ and (2) - containing $24^{*} 24=576$ matrices - has to be used. Surprisingly, the upper bound for the angles is again $62.8^{\circ} 3$. Obviously, the specimen symmetries in (2) do not act on the rotation angle. Indeed, it is easy to prove:

If the s sample symmetries are a sub-group of the 1 lattice symmetries, the $s 1$ matrices $O_{i k}$ break up into 1 classes of $s$ matrices each. The matrices within a class have all the same angle, which equals the angle of a suitably chosen $O_{i}$. This holds for all lattice symmetries.

Proof: $O_{i k}=L_{i} O S_{k}=S_{k}^{-1} S_{k} L_{i} O S_{k}=S_{k}^{-1} L_{j} O S_{k}=S_{k}^{-1} O_{j} S_{k}$.

There will in general be just one matrix with the smallest angle among the $O_{i}$, say $M$, but $s$ such matrices among the $O_{i k}$. They are obviously $\mathbf{M}_{k}=\mathbf{S}_{k}^{-1} \cdot \mathbf{M} \cdot \mathbf{S}_{k}$, having the axes $\mathbf{S}_{\mathbf{k}}^{-1} \cdot \underline{m}$. Thus, the axes of all $\mathbf{M}_{\mathbf{k}}$ are obtained by transforming one of them with all the sample symmetries. Obviously one and only one of these is found in the orientation triangle defined by the sample symmetries. Thus we have the result:

A unique representative can be selected out of the matrices (2) by requiring that the rotation angle is as small as possible and the rotation axis is in the orientation triangle defined by the sample symmetries. This holds for all lattice symmetries.

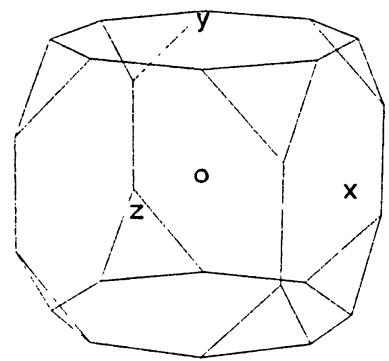

a)

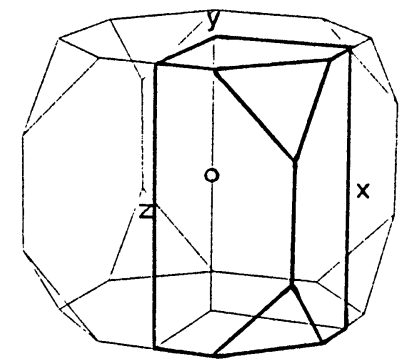

b)

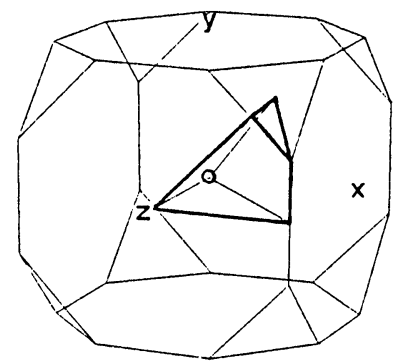

c)

Figure 1 Orientation spaces in the Rodrigues representation for cubic lattices: a) sample without symmetry b) ortho-rhombic sample c) cubic sample (disorientation).

To illustrate this, fig. 1 shows three orientation spaces for cubic lattices in the Rodrigues representation. The origin is marked by "O". All rotations are represented by points inside the polyhedra. The axis, $\underline{n}$, and the angle, $\omega$, of the orientation can be obtained from the radiusvector of the representing point via (1). The truncated cube in fig.la is the orientation space for full orientatiens, i.e. no sample symmetries. The shortest vectors reaching the surface are found along the axes $\tan (45 / 2)=\sqrt{2}-1$. The shortest radius vector along the [111] directions reaching the truncating triangles has a 
length of $\tan (60 / 2)=1 / 2$. The maximum angle (longest radius vector) is realized at the corners $\tan (62.8 / 2)=\sqrt{23-16 \sqrt{2}}=0.61$. The rectangular coordinates of one corner are $(\sqrt{2}-1, \sqrt{2}-1,3-\sqrt{8})$.

If the sample has some symmetries, the orientation space is that section of the truncated cube which is cut out by the orientation triangle of the sample symmetry. Fig.1c shows for cubic sample symmetry the well known Mackenzie cell in its most simple form - bounded by planes - a pleasant feature of the Rodrigues space. Fig. $1 \mathrm{~b}$ shows the must important orientation space, the one for ortho-rhombic sample symmetry. Again it is cut out of the truncated cube, but now according to the orientation "biangle" of the ortho-rhombic sample symmetry. This is the orientation space in which rolling textures of cubic materials can be presented.

\section{CHARACTERIZATION OF THE RODRIGUES SPACE AS THE MOST SIMPLE THREE-DIMENSIONAL ORIENTATION SPACE.}

In the following it will be shown, that the most natural representation of rotations is possible on the surface, $\mathrm{H}_{4}$, of the hyper-sphere in the fourdimensional space, $R_{4}$, in the sense that this is the only three-dimensional manifold with the required metric. Furthermore it will be shown that the Rodrigues space is the most natural representation in three-dimensional space since it is the geodesic projection of $H_{4}$ onto $R_{3}$, thus preserving most of the metric of $\mathbf{H}_{4}$.

The proper three-dimensional rotations are isomorphic to the threedimensional orthogonal matrices $A$ with $\operatorname{det}(A)=1$, which form a Lie group, $\mathrm{SO}(3)^{45}$. The representations of $\mathrm{SO}(3)$ have been studied extensively because of their far-reaching implications for quantum mechanics 67 . One central result is the recognition of the two-to-one homomorphic mapping of SU(2) onto $\mathrm{SO}(3)$. SU(2) is the group of the special $(\operatorname{det}(u)=1)$, unitary twodimensional complex matrices $u$, which may be written

$$
\mathbf{u}=\left(\begin{array}{cc}
u & v \\
-v^{*} & u^{*}
\end{array}\right) \quad \text { with } \operatorname{Re}(u)^{2}+\operatorname{Im}(u)^{2}+\operatorname{Re}(v)^{2}+\operatorname{Im}(v)^{2}=1
$$

The mapping is given explicitly by 6

$$
\mathbf{A}(\mathbf{u})=\left(\begin{array}{ccc}
\operatorname{Re}\left(u^{2}+v^{2}\right) & \operatorname{Im}\left(u^{2}-v^{2}\right) & 2 \operatorname{Im}(u v) \\
-\operatorname{Im}\left(u^{2}+v^{2}\right) & \operatorname{Re}\left(u^{2}-v^{2}\right) & 2 \operatorname{Re}(u v) \\
2 \operatorname{Im}\left(u v^{*}\right) & -2 \operatorname{Re}\left(u v^{*}\right) & u u^{*}-v v^{*}
\end{array}\right)
$$

Thus the two pairs of complex numbers $\pm(u, v)$ represent the rotation given in (5). They are called Cayley-Klein parameters.

A point, $X$, in the 4 -dimensional space, $R_{4}$, may be uniquely associated with u by

$$
X_{1}=\operatorname{Re}(u), \quad X_{2}=\operatorname{Im}(u), \quad X_{3}=\operatorname{Re}(v), X_{4}=\operatorname{Im}(v)
$$

Because of (4) we have $X^{2}=1$. Thus the pairs of opposing points, $\pm X$, on the surface of the unit hyper-sphere, $\mathrm{H}_{4}$, in $\mathrm{R}_{4}$ may be used as representatives of the rotations via $(6,5,4)^{8}$.

We define the vector-part, $\vec{X}$, of $\mathbf{X}$ as $\vec{X}=\left(X_{2}, X_{3}, X_{4}\right)$ or $\left.\mathrm{X}=\left(X_{1}, \vec{X}\right)\right)$. $\mathrm{X}_{1}$ and $\vec{X}$, are very simply related to the angle, $0^{\circ} \leq \omega<180^{\circ}$, and axis, $\underline{n}$, of the associated rotation $\mathbf{A}$ :

$$
X_{1}=\cos (\omega / 2), \quad \vec{X}=\sin (\omega / 2) \underline{n} \quad \text { or } \quad X=(\cos (\omega / 2), \sin (\omega / 2) \underline{n})(7)
$$


The consecutive execution of $\mathbf{B}$ and then $\mathbf{A}$, the multiplication of the rotations, is very simply reflected in the associated $\mathbf{Y}(\mathbf{B}), \mathbf{X}(\mathbf{A})$ and $Z(\mathbf{A B})$ :

$$
Z_{1}=X_{1} Y_{1}-\vec{X} \cdot \vec{Y}, \quad \vec{Z}=X_{1} \vec{Y}+Y_{1} \vec{X}+\vec{X} \times \vec{Y}
$$

As a consequence the invers of $X$ is obtained by changing the sign of $\vec{X}$. This agrees with (7). If we write $\mathbf{Z}=\mathbf{X Y}$ and interpret $\mathbf{X}, \mathbf{Y}, \mathbf{Z}$ as quaternions, then (8) is identical to the quaternion multiplication.

The distance between two rotations, $\mathbf{A}, \mathbf{B}$, is usually defined by some function of the angle, $\omega$, of the interconnecting rotation, $\mathbf{C}=\mathbf{A B}-1$, say

$d(A, B)=\omega\left(\mathrm{AB}^{-1}\right) / 2$. Due to $(7,8): \cos (\omega / 2)=Z_{1}=X_{1} Y_{1}+\vec{X} \cdot \vec{Y}(9)$ Thus the angle in $R_{4}$ between $X$ and $Y$ or - equivalently - the distance measured within the unit hyper-sphere $H_{4}$ between $X$ and $Y$ is $\omega / 2$. We thus have: The metric of rotations as defined by (9) is identical to the $\mathbf{R}_{\mathbf{4}}$-induced metric in $\mathbf{H}_{\mathbf{4}}$.

Since multiplication of two rotations by one given rotation preserves their distance, as defined by (9), the $\mathbf{R}_{\mathbf{4}}$-distances are also preserved. Thus we have:

1. $\mathrm{H}_{4}$ is rigidly rotated if all $X$ are multiplied with one given rotation 2. integration over $\mathrm{H}_{4}$ using the hyper-surface element $\mathrm{dH}_{4}$ is an invariant integration in the sense of Hurwitz.

From the above it is clear, that the most natural representation of rotations can be achieved in $\mathrm{H}_{4}$ with its $\mathrm{R}_{4}$-induced metric. However, $\mathrm{H}_{4}$ is noneuclidian with a constant positiv curvature. Therefore, although beeing a three-dimensional manifold, $\mathrm{H}_{4}$ cannot be projected into the euclidian $\mathrm{R}_{3}$ without deforming its metric. The problem is analogous to that of mapping the earth's surface onto a plane - the dimensionality beeing just raised by one. In both cases projections are available which preserve the geodesic lines or the volume, to name the most important ones. Here we have an additional requirement: Because of the importance of the axis of rotation, the projection $\underline{x}(\mathrm{X})$ should be parallel to it:

The geodesic projection

$$
\underline{x}(X)=f(\omega) \underline{n}
$$

$$
\underline{x}=\underline{X} / X_{1}
$$

yields $f(\omega)=\tan (\omega / 2)$, which is just the Rodrigues representation (1).

The functional determinant of the projection (10) can easily be shown to be

$$
d H_{4} / d V=\sin ^{2}(\omega / 2) /\left(2 f^{\prime}(\omega) f^{2}(\omega)\right)
$$

Inserting $f(\omega)=\tan (\omega / 2)$ into (12) yields

$$
d V=\left(1+\underline{n}^{2}\right)^{2} d H_{4}
$$

Conservation of the volume, $d H_{4}=d V$, is possible since (12) yields 2 $f(\omega)=\sqrt[3]{3(\omega-\sin \omega) / 4}$.

The geodesic Rodrigues projection (11) is by far the most convenient one since it transforms the geodesic lines of $H_{4}$ into the geodesic lines of $R_{3}$ : In general the geodesic line between two given points is the shortest line connecting them. In euclidian $\mathrm{R}_{3}$ it is a straight line, on $\mathrm{H}_{4}$ it is a hyper-circle with its center at the origin - in analogy to the great circles on a sphere in $\mathrm{R}_{3}$. Since $\mathrm{H}_{4}$ is non-euclidian with a constant positiv curvature, $\mathrm{H}_{4}$ cannot be projected into the euclidian $R_{3}$ without deforming its metric. In spite of that it can be projected such that all geodesics - in the case of cartography all meridians and great circles - become straight lines, thus preserving as much as possible of the original metric. 
In terms of rotations the geodesic lines in $\mathrm{H}_{4}$ can be characterized as follows:

$$
R(\omega)=A \cdot B(\omega, n), \quad-180^{\circ} \leq \omega \leq 180^{\circ} \text {. }
$$

where $B(\omega, n)$ is the set of rotations about the fixed axis $n$ with angle $\omega$. This is obviously identical to the definition of fiber textures. Thus we have: All fiber texture axes are represented by straight lines in the Rodrigues space. Furthermore the following is a direct consequence of the geodesic nature of the Rodrigues space and has been discussed in detail by Frank 1 in a different context: All bounding surfaces introduced by the symmetries are planes in the Rodrigues space. Both properties simplify calculations as well as visual perception. As shown above they are unique to the Rodrigues space because it is the geodesic projection of $\mathrm{H}_{4}$. Examples are given in fig. 3 and fig. 1 .

Since the projection is geodesic, it cannot simultaneously preserve the volume. However, this is not very detrimental because usually the region of interest is confined to the interior of the truncated cube of fig.la. There, for $0^{\circ} \leq \omega<62,8^{\circ}$, the invariant volume element deviates according to (13) from the euclidian volume element by less than a factor of two, which means for the linear dimensions of the volume element just $24 \%$.
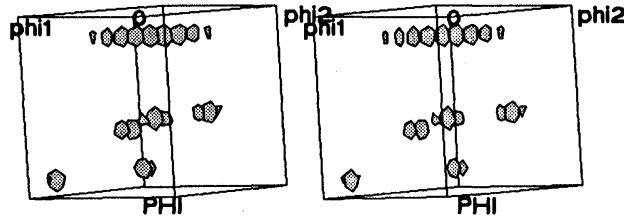

a)

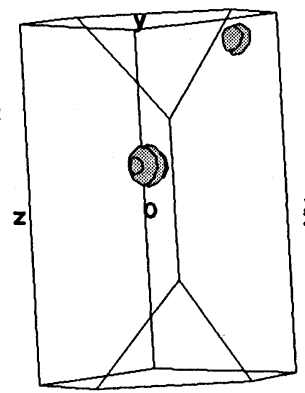

b)

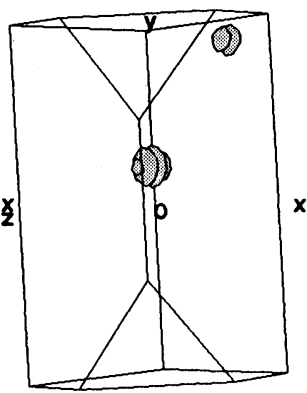

Figure $250 \%$ contour lines of two isolated orientations in Euler and Rodrigues representation presented in three dimensions as stereo pairs.

\section{CALCULATION OF ODF'S IN RODRIGUES SPACE} FROM EXISTING DATA IN EULER SPACE AND VICE VERSA.

Because of the convenient properties of the Rodrigues space we follow Frank 1 in recommending the latter for graphical representation of orientation distributions.

The transformation between the two spaces is given by

$$
\underline{r}=\tan \frac{\Phi}{2}\left(\frac{\cos \frac{\phi_{2}-\phi_{1}}{2}}{\cos \frac{\phi_{2}+\phi_{1}}{2}}, \frac{-\sin \frac{\phi_{2}-\phi_{1}}{2}}{\cos \frac{\phi_{2}+\phi_{1}}{2}}, \frac{\tan \frac{\phi_{2}+\phi_{1}}{2}}{\tan \frac{\phi}{2}}\right)
$$

\section{COMPARISON OF THE SUITABILITY OF THE EULER AND RODRIGUE SPACE FOR REPRESENTING ODF'S}

In order to compare the properties of the two spaces, an artifical orientation distribution is shown in fig. 2 in both spaces. To facilitate visual perception the plots are given as perspective stereo pairs of the $50 \%$ contours. The ODF consists of two Gaußian distributions of identical variance of $4.6^{\circ}$ centered around two different orientations. 
1. There are six spheres instead of two in the Euler space because of the threefold representation of each orientation in this space.

2. The bounding surfaces which separate the three symmetrically equivalent sub-cells of the Euler space (not shown in fig.2a) are curved, whereas the bounding surfaces of the Rodrigues space are plane.

3. The spheres strongly differ in size and shape in the Euler space although all spheres contain the same number of individual orientations and are isotropic. This is due to the fact that the ratio between invariant and euclidian volume element strongly deviates and even becomes zero at $\phi=0$. To alleviate this problem, not the orientation density, $\rho$, but the ratio $\rho / \rho_{\text {statistical }}$ is usually plotted in Euler space. This was also done in fig.2, still giving results, which are not satisfying. In the Rodrigues space $\rho$ may be plotted directly

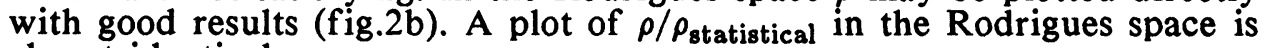
almost identical.

4. Fig. 3 demonstrates that fiber axes are curved in Euler space and straight in Rodrigues space.
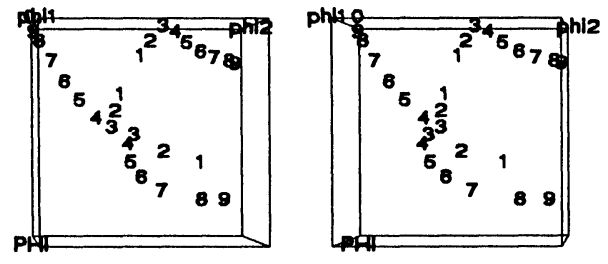

a)
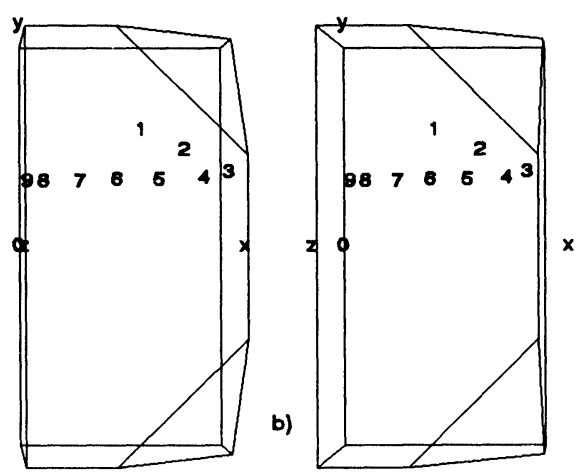

Figure 3 Some orientations within an ideally sharp fiber texture are plotted and labelled by consequtive numbers. Neighbouring orientations differ by $10^{\circ}$. The segmentation of the lines is due to the "Umklapp" effects of the symmetries.

\section{References}

1. F.C. Frank, Proc. of the ICOTOM 8 Conference, Santa Fee, USA, 1987, eds.J.S. Kallend and G. Gottstein (The Metallurg. Society, 1988), p. 3.

2. G. Ibe and K. Lücke, Texture, 1, 87 (1972).

3. J.K. Mackenzie, Biometrika 45, 229 (1958).

4. J. von Neumann, Mathematische Grundlagen der Quantenmechanik (Springer, Berlin, 1932).

5. J.F.Cornwell, Group Theory in Physics (Academic Press, London, 1984).

6. E.P. Wigner, Group Theory and its Application to the Quantum Mechanics of Atomic Spectra (Academic Press London, 1959).

7. B.L. van der Waerden, Die Gruppentheoretische Methode in der Quantenmechanik (Springer, Berlin, 1932).

8. R. Deltheil, Probabilités géométriques (Gauthier-Villars, Paris, 1926). 